УДК 336. 221; 332.14.

\title{
ПРОБЛЕМЫ И ОСОБЕННОСТИ ЭКОНОМИЧЕСКОГО АНАЛИЗА ИСПОЛЬЗОВАНИЯ ВТОРИЧНОГО СЫРЬЯ
}

\author{
Бочкова С.B. \\ к.э.н., доцент, кафедра Финансового менеджмента \\ Ровенская А.A. \\ Мишко Ю.А. \\ Институт экономики и финансов ФГБОУ ВО «Сыктывкарский \\ государственный университет им. Питирима Сорокина»
}

\begin{abstract}
Аннотация: Материальные затраты составляют значительную долю всех затрат на производство продукции, работ, услуг. Рациональное использование материальных ресурсов - один из важнейших факторов роста объема продаж и снижения себестоимости продукции, а, следовательно, и роста прибыли и рентабельности. Удовлетворение потребности предприятия в материальных ресурсах осуществляется двумя путями: экстенсивным и интенсивным. Экстенсивный путь предполагает увеличение добычи и производства материальных ресурсов и связан с дополнительными затратами. Интенсивный путь удовлетворения потребности предприятия в материалах, сырье, топливе и других материальных ресурсах предусматривает более экономное расходование имеющихся запасов в процессе производства продукции. Используя достижения науки, технологический прогресс может быть организован таким образом, чтобы отходы производства вновь поступали в производственный цикл как вторичное сырьё.
\end{abstract}

Ключевые слова: вторичное сырье, эффективность, использование вторсырья, методы анализа, отходы производства.

\section{PROBLEMS AND FEATURES OF ECONOMIC ANALYSIS OF THE USE OF SECONDARY RAW MATERIALS}

\section{Bochkova S.V. Rovenskaya A.A. Mishko Y.A.}

\footnotetext{
Abstract: Material costs make up a significant share of all costs for the production of products, works, and services. Rational use of material resources is one of the most important factors for increasing sales and reducing the cost of 
production, and, consequently, increasing profits and profitability. There are two ways to meet the company's demand for material resources: extensive and intensive. The extensive path involves increasing the production and production of material resources and is associated with additional costs. The intensive way to meet the needs of the enterprise in materials, raw materials, fuel and other material resources provides for more economical use of existing stocks in the production process. Using the achievements of science, technological progress can be organized in such a way that production waste re-enters the production cycle as secondary raw materials.

Key words: secondary raw materials, efficiency, use of recyclable materials, methods of analysis, production waste.

В настоящий момент за рубежом в промышленности в сфере переработки отходов, сформировался большой опыт, разработаны и реализованы множественное количество технологических проектов по переработке различных видов вторичного сырья. Однако в России на промышленных предприятиях учет отходов фактически отсутствует, а существующий в отдельных отраслях промышленности не содержит достоверных сведений об объемах образования этих ресурсов. Относительно зарубежных предприятий, где используется от 60 до $95 \%$ вторсырья, на отечественных, приблизительно, 50 \% используется как вторичное сырьё. Остальная часть погибает, тем самым снижая эффективность деятельности предприятий. Такое бесчисленное количество отходов оказывает отрицательное влияние на себестоимость продукции. Удельный вес издержек производства на вторичное сырьё в себестоимости изделий и материалов достигает 60 - $80 \%$ [1].

Это приводит к тому, что полностью теряется контроль за сохранностью и правильным отражением движения отходов по производственным подразделениям. Однако их величина непостоянна, она зависит от того, насколько прогрессивны нормы расхода материала, от уровня применяемой технологии и особенностей производства.

В соответствии с ГОСТом 25916-83 под вторичным сырьем понимаются «вторичные материальные ресурсы, которые в настоящее время могут повторно использоваться в народном хозяйстве».

Для регулирования использования вторичного сырья разработана государственная политика России, которая должна предусматривать 
промышленный подход к организации сбора и использования отходов в качестве вторичного сырья, опираясь на формирующиеся мировые тенденции и сформировавшийся собственный опыт [2].

Организация производства на предприятиях призвана обеспечить максимальный выход основных видов продукции, предупреждение брака и наиболее допустимую переработку отходов. Внимание должно быть сконцентрировано на использовании вторичного сырья, которое может быть использовано в другом производственном процессе. Достижение этой цели обеспечивается современными технологиями, которые создают возможность эффективно перерабатывать вторичное сырье производства для их повторного использования в технологических процессах. Возможность использования вторичного сырья имеет два основных варианта:

А) Использовать производственные отходы повторно для изготовления некоторых видов продукции и материалов, что значительно увеличит выручку от реализации;

Б) Реализовывать отходы производства в качестве сырья другим хозяйствующим субъектам по сниженной цене, то есть цене возможного использования.

Анализ отходов производства начинается с определения показателей материалоотдачи и материалоёмкости продукции:

1. Материалоотдача показывает какое количество продукции приходится на 1 рубль материальных затрат для компании. Так, чем выше данный показатель в динамике, тем более эффективно производство:

$$
\mathrm{Mo}=\mathrm{V} / \mathrm{M} \text {, }
$$

где $\mathrm{V}$ - объем реализованной продукции,

M - материальные затраты.

Материалоёмкость является обратным показателем материалоотдачи и показывает в какое количество материальных затрат предприятию обходится 1 рубль произведенной продукции:

$$
\mathrm{Me}=\mathrm{M} / \mathrm{V}
$$

Следовательно, убывающее значение данного показателя является положительным признаком для предприятия.

Значения материалоотдачи и материалоемкости рассчитываются по плану и по факту, определяется отклонение. По окончании расчета дается оценка результата. 
2. Теперь необходимо проанализировать изменение запланированной эффективности использования материалов. Так, рассчитывается коэффициент равный отношению стоимости всех используемых материалов к производственным отходам:

$$
\text { К исп. м. = См / Co, }
$$

где См - стоимость материальных затрат,

Со - стоимость отходов.

Данный коэффициент характеризует во сколько раз стоимость материалов (по цене исходного сырья) превышает стоимость отходов (по цене возможного использования).

3. Третий этап предусматривает расчет стоимости отходов по запланированному значению (плановый норматив отходов) и по фактическому. Для этого коэффициент эффективности использования материалов отдельно умножают на возвратные отходы по плану, затем по факту соответственно:

$$
\text { Со }=\text { Во * Кисп.м., }
$$

где Во - возвратные отходы.

В результате полученные значения сравнивают, дают оценку и определяют изменение стоимости производственных отходов.

4. Если предприятие реализует все отходы, то определяется коэффициент полезного использования материалов (отношение разности стоимости материалов и стоимости отходов к стоимости материалов) по плану и факту, его отклонение:

Коэффициент полезного использования материалов определяется как в:

$$
\text { Кп.исп.м. }=(\mathrm{Cm}-\mathrm{Co}) / \mathrm{CM}
$$

После рассчитывается изменение доли отходов производства:

$$
\text { Ко }=100 \% \text { - Кп.исп.м. }
$$

5. По итогам анализа определяют, как влияет изменение величины отходов на изменение объема выпуска, то есть рассчитывают отношение изменение величины отходов к плановой материалоёмкости [3].

Данный анализ отходов производства позволяет выявить наличие сверхплановых отходов и сделать выводы о снижении объема выпуска продукции или, наоборот, его увеличении, а также определить дальнейшие меры по снижению материалоемкости и величины планового норматива.

При подготовке нормативов по образованию отходов производства необходимо руководствоваться Федеральным законом "Об отходах 
производства и потребления" от 24.06.1998 N 89-Ф3, методическими рекомендациями. [2]

Методические рекомендации по оценке объемов образования отходов производства и потребления включают различные методы и методики исследования оценки образования отходов, формулы расчета и справочные данные для такой оценки, рекомендации по их выбору в зависимости от вида отходов.

Методика анализа использования отходов выделяет четыре основных способа оценки объема образования отходов:

- прямой расчет на основе данных материального баланса использования в конкретном технологическом процессе (или производстве) исходного сырья и получения продукции;

- расчет при помощи использования удельных показателей (или нормативов) образования отходов;

- определение объема образования отходов на основе бухгалтерской отчетности и других статических данных по плану и факту за прошлые периоды;

- расчет по формулам, составленным на основе данных конструкторской и технологической документации и регламентов на изготовление продукции.

Несмотря на разность подходов к определению производственных отходов, все они опираются на единую методологию:

- определение источников образования отходов;

- выявление перечня образующихся отходов;

- изучение и анализ отчетных данных прошлых периодов;

- определение удельных показателей образования отходов, учитывая применяемые технологии;

- непосредственный расчет объемов образования отходов по имеющимся формулам с использованием статистических и справочных данных.

Далее на основании методических рекомендаций происходит выбор метода, по которому будет происходить оценка показателей образования отходов. Этот выбор определяется поставленными целями и задачами, а также рядом факторов: вид объекта (хозяйствующий субъект, регион, отрасль, муниципальное образование), наличие и количество исходных данных по производству, необходимая степень точности оценивания. Например, для 
оценки объемов образования отходов производства какой-либо отрасли целесообразнее будет применять метод оценки по удельным показателям. Для предприятия же предпочтительным вариантом чаще всего будет оценка объемов образования того же отхода на основе расчетно-параметрического метода, как наиболее точного и конкретного [4].

Итак, каждый из названных подходов предполагает использование двух или более методов оценки объемов образования отходов производства:

- метод оценки на основе данных материально-сырьевого баланса;

- метод оценки по удельным показателям образования отходов;

- метод оценки по среднестатистическим данным образования отходов;

- экспериментальный метод;

- расчетно-параметрический метод.

Теперь более подробно рассмотрим основные методы оценки образования отходов:

1. Метод оценки на основе данных материально-сырьевого баланса

Метод материально - сырьевой баланса является основным, базовым при нормировании образования отходов производства и отличатся особой точностью. Но несмотря на это имеет ограниченное применение. Это происходит потому, что для его расчета необходима большая номенклатура исходных данных. Исходные и расчетные данные формируются в виде таблицы или нескольких таблиц (указанных согласно образцу в приложении методических указаний) и включают в себя:

- количество используемого сырья и материалов в единицу времени;

- количество сырья и материалов, перешедшее в продукцию;

- количество произведенной продукции;

- нормы естественной убыли;

- количество веществ, выбрасываемых в атмосферный воздух и сбрасываемых со сточными водами.

Норматив отходов рассчитывается по фактическому расходу материалов и является базовым для производственных предприятий. В качестве исходных данных для расчета используется внутренняя документация предприятия, регламентирующая использование сырья и материалов, технологические карты и регламенты, данные по учету расхода материалов и сырья, получения продукции, источники образования отходов и выбросов. 
В зависимости от имеющегося набора исходных данных расчет нормативов образования отходов может производиться по нескольким вариантам. Но в общем виде формула выглядит следующим образом: объем образующихся в технологическом процессе отходов равняется разности между количеством потребленного сырья и количеством произведенной продукции с учетом неизбежных безвозвратных потерь.

Таким образом, метод в основном используется именно крупными промышленными предприятиями, где традиционно применяется материальносырьевой баланс. Это относится к предприятию таких отраслей, как: пищевая промышленность, лесоперерабатывающее и химическое производство, черная и цветная металлургия, энергетика и др.

2. Метод оценки по удельным показателям образования отходов

Данный метод целесообразней всего применять по регионам, муниципальным образованиям или отрасли в целом для укрупненной оценки образования отходов, так как в некоторых случаях отличается достаточно широким колебанием значений удельных показателей (иногда верхний от нижнего отличается на порядок). Прежде всего он удобен и предназначен для органов природоохранной деятельности и органов административнохозяйственной деятельности, проводящих экспресс-оценку по образованию объемов отходов, их обращению, экологическому контролю и лимитам размещения. Метод рекомендуется к использованию в тех отраслях, где он считается традиционным для определения образования отходов. Сюда относится транспорт, строительство, жилищно-коммунальное хозяйство и т.д. [5]

При применении данного метода используются отраслевые нормативы образования отходов, а также нормативы, указанные в «Сборнике удельных показателей образования отходов производства и потребления» (1999 г.). Однако из-за некоторых устаревших данных значения этого сборника при необходимости корректируются в связи с модернизацией технологий на производстве.

3. Метод оценки по среднестатистическим данным образования отходов Данный метод не требует глубокого, комплексного подхода в изучении, трудных методологических расчетов и специального оборудования. Именно поэтому первоначально был широко распространён и применялся на разных типах производственных предприятий. В современных условиях метод применяют только в том случае, если невозможно применить другие методы 
расчета из-за отсутствия определённых исходных данных. Другим случаем использования метода является его базовое назначение, что означает его использование в качестве опоры для других методов расчета или же его комбинирование для более полной картины анализа производства.

Исходные данные для расчета получают на основе анализа отчетностатистических данных (например, указанное в бухгалтерском отчете списание упаковки, тары, спецодежды, малоценных средств и др.), а также на опытно-производственных показателях, которые могут быть получены с помощью прямого измерения (объем, масса и др.) за определенный промежуток времени. Обычно необходимый интервал времени составляет 3 и единиц времени (месяцев, кварталов, лет).

Также необходимо учитывать сопряженные с фактическими данными факторы, оказывающие непосредственное влияние на образование отходов: применяемые материалы, развитие технологий, износ оборудования и т.д. Расчет может включать неограниченное количество факторов и параметров. Возможно присутствие и специфических коэффициентов для определенных видов продукции и сырья (обводненность или, наоборот, усушка, загрязненность нефтепродуктами и др.).

Таким образом, метод считают ограниченным, временным, недостаточно точным и зачастую применяют в совокупности с другими расчетами.

4. Экспериментальный метод

Экспериментальный метод очень схож с методом оценки по среднестатистическим данным образования отходов и также чаще всего рассматривается только в ограниченных ситуациях, чаще всего как временный. Его использование целесообразно в следующих случаях:

- при освоении новых видов технологий и производств;

- при ярко выраженном переменном характере образования отходов;

- при выполнении определенного вида работ, несвойственных для данного предприятия;

- при образовании отходов, которые были получены при выполнении работ в экстремальных условиях, маловероятных в будущих периодах.

Метод основан на проведении фактических опытных измерениях, а результатом являются полученные нормативы расходов, которые используются предприятием лишь в определенные промежутки времени и нестандартных условиях. Обычно предприятия используют его разово, при 
невозможности использования для расчета других методов, например, расчетно-параметрического из-за большой трудоёмкости и нехватки данных.

5. Расчетно-параметрический метод

Расчетно-параметрический метод является, пожалуй, самым универсальным из всех перечисленных, характеризуется высокой точностью и практически неограниченным перечнем отходов. Исключением из перечня являются только радиоактивные отходы, регулируемые специальными документами [4].

Главным преимуществом является индивидуальный подход к расчету объема образования отходов на каждом конкретном предприятии. Эта особенность метода подразумевает то, что организация может разработать некоторые нужные расчетные формулы самостоятельно. При этом необходимо учитывать, что в этом случае они должны быть согласованы с органами природоохранной деятельности.

Метод в особенности рекомендуют применять в том случае, если образующиеся отходы формируются непосредственно на данном предприятии. Поскольку он обладает высокой степенью точности, то предусматривает большой объем исходных данных. Данные берутся на основании анализа отчетно-статистических материалов, технологической документации, нормативных величин, а также из самого положения методических рекомендаций. Если же остаются недостающие данные, то они замеряется экспериментальным методом, фактическими измерениями.

Расчет по этому методу предусматривает использование специальных формул, содержащих показатели и коэффициенты, наиболее полно отражающие фактическое состояние отхода в части количественной оценки вещественно-материального состава.

Таким образом, метод считается наиболее полным, точным, технически и экономически обоснованным.

Учитывая вышеизложенное, представляется целесообразным гибкое использование различных методов, с определением приоритетов тех или иных методов для определенных видов отходов, хотя для оценки объема образования конкретного отхода в различных ситуациях возможно применение различных методов.

Итак, как было сказано выше, любой технологический процесс создания продукции, работ и услуг предусматривает производственные потери в виде отходов. Однако их величина зависит от множества факторов: уровня 
применяемых технологий, особенностей производства, нормы расхода материалов и их прогрессивности. На любом производстве целесообразно стремиться к снижению производственных отходов и применять малоотходные (в идеале безотходные технологии), а также новейшие прогрессивные материалы или материалы с заданными свойствами.

\section{Список литературы}

1. Коковкин А.В. Проблемы в сфере обращения с отходами производства и потребления на Европейском Северо-Востоке России и пути их решения. Материалы всероссийской научной конференции ч.2 2018.

2. Федеральный закон "Об отходах производства и потребления" от 24.06.1998 N 89-Ф3 [Электронный ресурс]. URL: http://www.consultant.ru/ document/cons_doc_LAW_19109/ (дата обращения: 05.10.2020).

3. Мирзоева А.Р., Шогенова М.Х. Оценка экономической эффективности комплексного использования сырья // Экономический анализ: теория и практика. 2019. №33 (336). URL: https://cyberleninka.ru/article/n/ otsenka-ekonomicheskoy-effektivnosti-kompleksnogo-ispolzovaniya-syrya (дата обращения: 05.10.2020).

4. Девяткин В.В. Методические рекомендации подготовлены Государственным учреждением Научно-исследовательский центр по проблемам управления ресурсосбережением и отходами.

5. Врублевский Н.Д. Расчет эффективности использования м учет движения отходов производства. Профессиональный журнал для бухгалтера №12 2018. 